\title{
Integrating Volunteered Geographic Information into Pervasive Health Computing Applications
}

\author{
Peter Mooney \\ Geotechnologies Research Group, \\ Department of Computer Science, \\ National University of Ireland Maynooth, \\ Co. Kildare. Ireland. \\ Email: peter.mooney@nuim.ie
}

\author{
Padraig Corcoran \\ Geotechnologies Research Group, \\ Department of Computer Science, \\ National University of Ireland Maynooth, \\ Co. Kildare. Ireland. \\ Email: padraig.corcoran@nuim.ie
}

\begin{abstract}
In this paper we describe the potential for using Volunteered Geographic Information (VGI) in pervasive health computing. We use the OpenStreetMap project as a case-study of a successful VGI project and investigate how it can be expanded and used as a source of spatial information for pervasive computing technologies particularly in the area of access to information on healthcare services.
\end{abstract}

\section{INTRODUCTION}

Meaningful expansion of data access for researchers and other users is needed to improve the public's health [1]. Conway and van Lare [1] cite three serious issues preventing this expansion: cost and timeliness of data access, tiered access models, and aggregating diverse health data resources in usable forms [1]. This paper will show that ideas, conceptual models, and motivations from the Volunteered Geographic Information (VGI) community, when combined with pervasive computing technologies, could yield significant advances towards reduction or eventual elimination of these issues. "Pervasive computing technology" is in itself not a welldefined technology but more like a multidisciplinary research agenda involving technologically oriented research on things like hardware, communication technology, embedded hardware and software, software infrastructures, sensor technology, distributed computing, computer-supported cooperative work, human computer interfaces, sociological studies of the use of technology, etc. [2]. It is not a software product that one can buy off-the-shelf. Pervasive healthcare may then be defined from two perspectives: i) as the application of pervasive computing technologies for healthcare, and ii) as making healthcare available everywhere, anytime and to anyone. This paper concentrates on a crossover between these perspectives. The rest of this paper is organised as follows. The paper opens with section II and a description of the VGI community and how it works. Section III discusses both open access to data and information and open source software (OSS) in the domain of health informatics. A brief discussion is also provided of the next generation web development with HTML5. In section IV an overview of how healthcare services, locations, etc are represented, stored, and contributed to OpenStreetMap is provided. This section also illustrates an example of how easily any citizen can provide VGI by using OpenStreetMap, Flickr,
Foursquare, and Twitter. Section V outlines a summary of the potential advantages and disadvantages of integrating VGI into the data/information flows of pervasive health applications. This is necessary to allow experts and policy makers from outside the GIS/VGI domain to understand the strengths and weakness of VGI. Section VI is the final section of the paper and it provides a discussion of some concluding remarks and directions for future work.

\section{THE VGI COMMUNiTy}

The VGI community is a global crowdsourced (many volunteers working together) community which shares many similarities with the Wikipedia model of information collection. The OpenStreetMap project [3] is a crowdsourced geospatial database with volunteers all over the world. It is probably the most famous VGI project with a mission to create a free, constantly updating and improving, editable map of the world in addition to providing free access to the underlying spatial data (geometry and attributes). Masses of contributors from around the world are volunteering their time and efforts to collaboratively create a detailed base map. Many other volunteers in OpenStreetMap are working on: software development for OpenStreetMap, maintaining the OpenStreetMap Wiki website, organising mapping party events, etc. The growing spatial coverage, and high-quality content, has branched beyond "the converted" and has gained enthusiastic endorsement from the likes of Yahoo, ESRI, MapQuest [4], and Microsoft [5]. Other examples of less coordinated VGI include: open interfaces to exploit Twitter [6] where tweets are geo-located; open interface to online digital photo repositories such as Flickr [7] where photographic images are also geolocated; GeoNames [8] - a project to create a global database of city, town, village, and street names. These interfaces have been used successfully by researchers. For example, Lampos and Cristianini [9] collected 160,000 tweets on a daily basis for 24 weeks in the UK in an attempt to use social media generated information as a means of tracking public health incidents. Hollenstein and Purves [10] show results of harvesting georeferenced and tagged metadata associated with 8 million Flickr images and draw conclusions on how large numbers of people name and describe downtown city 
areas. The results of this work could be applied to analysis of tagging of photographs of natural or environmental disasters or incidents relating to public health such as forest fires or industrial accidents. In the next section we summarise how the VGI community has grown and introduce more details about OpenStreetMap.

\section{A. How the VGI community works}

VGI has built upon the rapid success of user-generated content on the Internet. In the last decade the Internet has entered a leading role to facilitate communication across borders, becoming more and more important as a source of communication, education, research and collaboration. The healthcare environment is no different and the penetration of the Internet into the healthcare setting has been profound and has changed professional collaboration and development [11]. Social media websites, such as YouTube, Facebook, MySpace, Twitter, etc are rapidly emerging as popular sources of health information especially for teens and young adults. Social media carries the advantages of low cost, rapid transmission through a wide community, and user interaction. Disadvantages include blind authorship, lack of source citation, and presentation of opinion as fact [12]. Despite these disadvantages the social acceptance of these websites cannot be underestimated. The health informatics model consists of three essential parts: data, information and knowledge. These elements are arranged in a hierarchy, with data at the base of the model providing the basis for establishing information and leading in turn to the potential generation of knowledge [13]. In this paper we show how VGI (with specific emphasis on OpenStreetMap) could be used as a spatial data component in a global health informatics setting. The crowdsourced approach of OpenStreetMap derives its success from citizens mapping and collecting data and information about their locality. Features being mapped include the location of garbage cans, pedestrian crossings, land cover types, shops, education facilities, to government buildings, roads and river networks. We explore how OpenStreetMap volunteers could map and collect information about healthcare facilities. All data in the OpenStreetMap database can be downloaded for free in a variety of spatial data formats. Additionally a number of open source tools are available to process this data and produce other formats such as Google Earth-friendly KML. Ormeling [14] argues that OpenStreetMap provides an unprecedented opportunity for anyone to "create the maps that they want when they want". A core motivation behind the production of VGI is likely the inaccessibility and cost of accurate sources of geographic information [15], [16]. The capacity of people from around the world to create geographic information has further been assisted by the drop in the price of GPS units and the wide availability of computers and smart mobile devices [15], [16].

\section{The 'Open' In HeAlth Informatics}

There is an increasing demand for more and better data in the area of healthcare [17]. This increase in demand has been driven by a number of factors including: advances in information technology, the development of new health information systems, and the increasing use of tools such as geographical information systems (GIS's) in healthcare informatics etc. Effective public health practice relies on the availability of public health data sources and assessment tools to convey information to investigators, practitioners, policy makers, and the general public. Emerging communication technologies on the Internet can deliver all components of the "who, what, when, and where" quartet more quickly than ever with a potentially higher level of quality and assurance, using new analysis and visualization tools. Open-source software provides the opportunity to build low-cost information systems allowing health departments with modest resources access to modern data analysis and visualization tools [18]. As public health awareness and interventions move beyond local, state and national boundaries towards a global health perspective, an increasing amount of public health data will need to be integrated and publicly accessible [18]. Open Health Informatics relies on the use of open interfaces so that every component of a software solution can be plugged in and out at will. This enables a 'best of breed' approach to open source and eliminating once and for all the product-centric culture.

\section{A. Open Source Approaches}

Several studies have investigated the breadth and diversity of Open Source Software (OSS) applications in health and medical informatics. While OSS adoption has potential, more research is needed to understand the nuances of OSS development and the cultural and technological barriers to adoption, particularly in the typical U.S. [19]. The vast majority of applications are also in English. The authors mark this as a serious disadvantage with a very small number of multilingual interfaces. Many of the problems faced by VGI when compared with traditional sources of spatial data/information are reflected in the use of OSS in health and medical informatics. Janamanchi et al [19] stress that one "cannot ignore the sizable barriers that OSS faces in the health care industry". McDonald et al [20] agree and state that the health and medical informatics industry is one "where almost all software in use is proprietary, the health information technology leadership tends to be conservative, and senior management has been eliminating the in-house, technical and developmental teams that could best leverage open-source software to the advantage of the institution". However they comment that the open source movement is changing the situation. Due to the nature of this movement, the sharing of knowledge and access to new development and refined and enhanced functionality in webbased applications, the healthcare informatics scene is "going through a major change". This provides encouragement that VGI could be a successful component in pervasive health computing in the health and medical informatics environment. Ideally all countries in the world should have sufficient resources to prepare for disasters, public health emergencies, etc and that there would be intrinsic, morally-driven motivation to produce national-level spatial data and mapping products and 
then the motivation to keep them up-to-date. In developing nations, where resources are already over-stretched, preparing for events that might potentially happen in the future receives lower priority.

\section{B. Preparing for the Next generation Web}

The concepts and philosphy of open access and open source are being built into the specifications for the next generation web. Mapping health information is one area of health informatics which could potentially undergo the greatest changes. Spatial health data is now seen as a valuable resource in the field of health and medicine. Chronic diseases such as cancer can display significant spatial pattern [21]. Consequentially spatial data can allow researchers to evaluate hypotheses about geographic variation, such as clustering, and to formulate new hypotheses. Disease maps offer a visual means of identifying cause and effect relationships existing between humans and their environment [22]. The Internet has emerged as an efficient way to share spatial health information through the use of web-GIS [23]. The ultimate ambition of any health web-GIS system is to provide the ability to both visualize and perform analysis of such data. Unfortunately due to limitations of the current version of HTML (the language used to represent web-pages) this introduces a trade-off between reducing functionality or providing this functionality through the installation of client plug-ins. For example, the current version of HTML does not provide any functional to render (visualize) or provide user interaction with geographical data. To overcome this issue many services pre-render the data on the server and transmit the corresponding images to the client. This is a valid solution but does not allow the client to perform a visualization adapted to their needs or interact with the data [23]. To overcome such limitations MacEachren et al. [24] proposes to use additional client plug-ins in the form of Adobe Flash. Although such plug-ins provide the required functionality they reduce the Web's openness and platform independence, and tend to lock users to specific technologies and vendors. The forth coming version of HTML, known as HTML5, hopes to reduce this trade-off through the introduction of many new API's (Application Programming Interface) which will provide the functionality which is lacking in the current version. In fact, Ian Hickson, one of the W3C's HTML5 editors, states that "One of our goals is to move the web away from proprietary technologies".

\section{WORKING WITH VGI DATA AND INFORMATION}

In this section we provide examples of how OpenStreetMap and other sources of VGI could be used in a health informatics setting. It is not possible to provide a beginners guide to using OpenStreetMap here. However a very good tutorial is available on the OpenStreetMap wikie [25]. Figure 1 shows a screenshot of the very popular JOSM (Java based editor for OpenStreetMap) [26]. In the screenshot a hospital polygon is undergoing a user edit. The user can easily edit the location or shape of the polygon, add or edit tagging information to the polygon, add icons etc. All of these edits can be done in

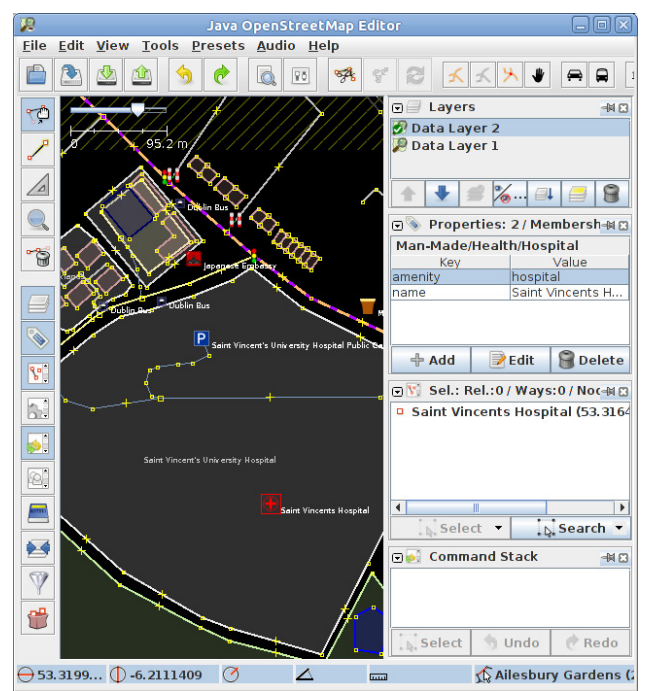

Fig. 1. Editing of a hospital polygon and point geographic feature in the JOSM (Java for OSM) graphical editor for OpenStreetMap

TABLE I

THE USAGE OF HEALTH INFORMATION AND SERVICE TAGS IN THE GLOBAL OPENSTREETMAP DATABASE

\begin{tabular}{|l|c|c|c|c|}
\hline Theme & Total & Points & Polygon & Relations \\
\hline Emergency & 6403 & 509 & 5880 & 14 \\
\hline Dentist & 9461 & 8976 & 484 & 1 \\
\hline Hospital & 59912 & 48033 & 11719 & 160 \\
\hline Pharmacy & 49719 & 47978 & 1740 & 1 \\
\hline Doctor & 12665 & 10404 & 2249 & 12 \\
\hline Healthcare & 967 & 840 & 126 & 1 \\
\hline
\end{tabular}

"offline" mode until the user is happy to submit these edited contributions back to the global OpenStreetMap database. JOSM supports a wide range of functionality. One particularly useful feature is the provision of functionality to allow users to upload GPX tracks from their GPS device. These tracks are then automatically overlayed onto the OpenStreetMap data for that region the GPX track was recorded in. The user can then edit these tracks and easily integrate them into the OpenStreetMap database. JOSM allows users to select a region from a simple map. Upon selection the JOSM software retrieves the spatial data from the OSM database as well as loading and editing existing nodes, ways, metadata tags and relations from the OSM database. At all times a conflict manager is running within JOSM which observes if the user is editing data incorrectly, adding illegal values to tags, etc.

In Table I the usage of health information and service tags in OSM is outlined. The information was extracted from the TAGINFO database in January 2011. The TAGINFO service [27] is a near-real-time tag query tool for the global OSM database. The "amenity=healthcare" is a composite tag for spatial objects and the OSM Map Features guidance page indicates that this can be used to identify the location of: offices for physicians, doctors, dentists, alternative medicine, nursing homes, etc. Analysis of the "amenity=hospital" shows 


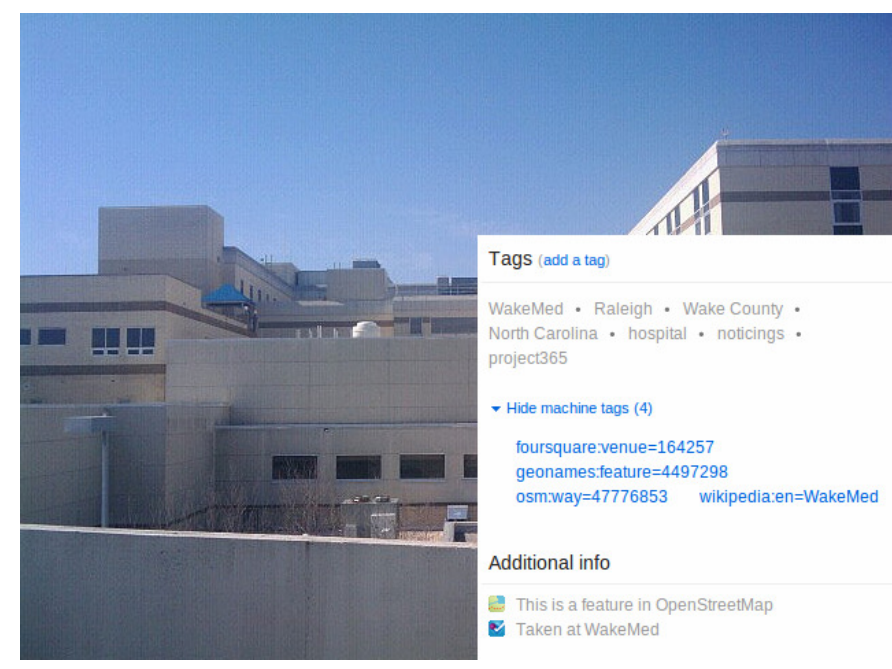

Fig. 2. A WakeMed healthcare facility in Raleigh, NC. USA. This photograph on Flickr is automatically linked to the OpenStreetMap database and to the social networking website FourSquare using simple text tags with the photograph

that OSM contributors are eager to include this information in the OSM database. Amongst the large set of possible values that the "amenity" key can take the value hospital is ranked $12^{\text {th }}$ most popular. The TAGINFO database does not require any special API or tools for access. Information is extracted from the global OSM database and presented in "drill-down" table format.

In Figure 2 a photograph from Flickr is shown (http: //www.flickr.com/photos/rharrison/4398781892/). At the bottom right corner of the picture 4 machine readable tags are provided. When the owner of this photograph uploaded this photo they included these tags in the upload information. The photograph is from a WakeMed Health facility in Raleigh, North Carolina. One of the tags osm: way $=47776853$ refers to the actual spatial data representation of this healthcare facility in OpenStreetMap (http://www.openstreetmap.org/?way= 47776853). This is highlighted in the web-based map in Figure 3. The key advantage to this approach is that the citizen who uploaded to Flickr need only know very basic information about OpenStreetMap. To find the identification number for the WakeMed facility, as represented in OpenStreetMap, they would have performed the following steps. First they would have visited OpenStreetMap.org and zoomed the map to the approximate location in Raleigh, NC where the facility is located. The second step involved click on the "Data" tab in the options pane for the map. When this option is activated the OpenStreetMap map automatically generates a new map clickable map layer which highlights all polygons, polylines, and points within the map window. The final step is where the building or object of interested is identified and clicked on. The metadata (or spatial information) about that object is displayed in a separate information pane from which the user can visually extract the osm-id corresponding to that building or object of interested. The special OpenStreetMap

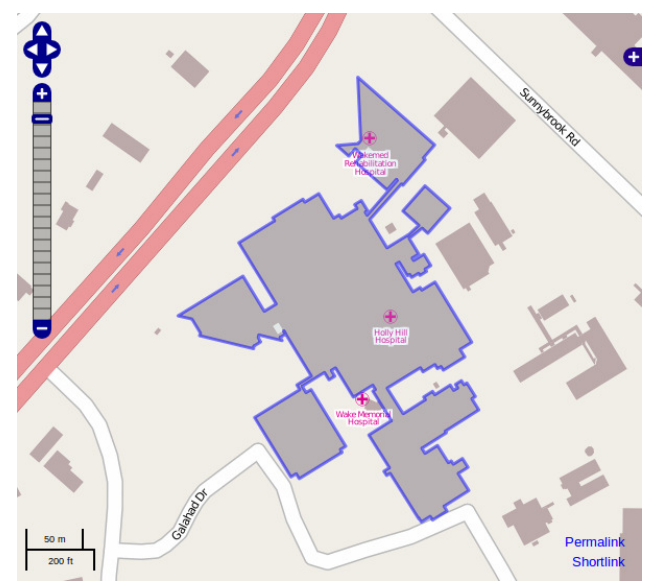

Fig. 3. Editing of a hospital polygon and point geographic feature in the JOSM (Java for OSM) graphical editor for OpenStreetMap

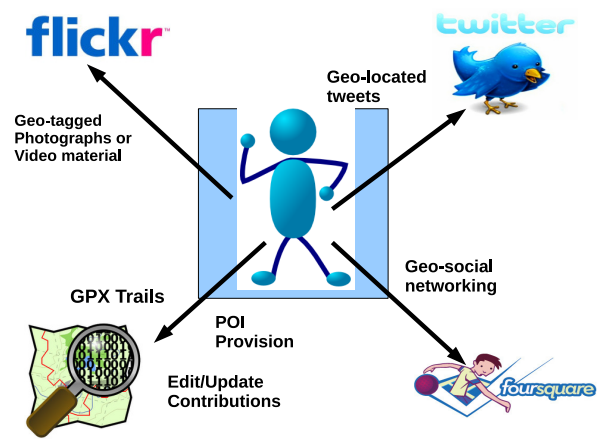

Fig. 4. This flowchart shows the user as the key generator of spatial content. This content has geolocation embedded intrinsically

tag for Flickr is created in the same simple way that tagging is performed in Flickr for any photograph.

\section{A. The citizen as a content generator}

In Figure 4 the user is the content generator. Using their mobile device or their desktop/laptop they can easily generate content which is inherently spatial. As a registered user of Twitter they can opt-in to having their messages (Tweets) annotated with their exact spatial locations. Flickr is able to store longitude and latitude data of the uploaded photographs. As the number of mobile phones and cameras which feature an integrated GPS increase this location information can be automatically embedded in the photograph image file. Users can manually locate in a map the place where the photo was taken from the Flickr website. The timestamp of each photograph is also stored along with any tagging information the user provides. As described in the previous section these photographs can then be linked to OSM features very easily. The Foursquare social network game is inherently geo-located. The Foursquare API [28] gives developers access to all of the data used by the Foursquare mobile applications. Developers can access the Foursquare checking-ins (where people reported that they were at a given time) and leverage uniquely 


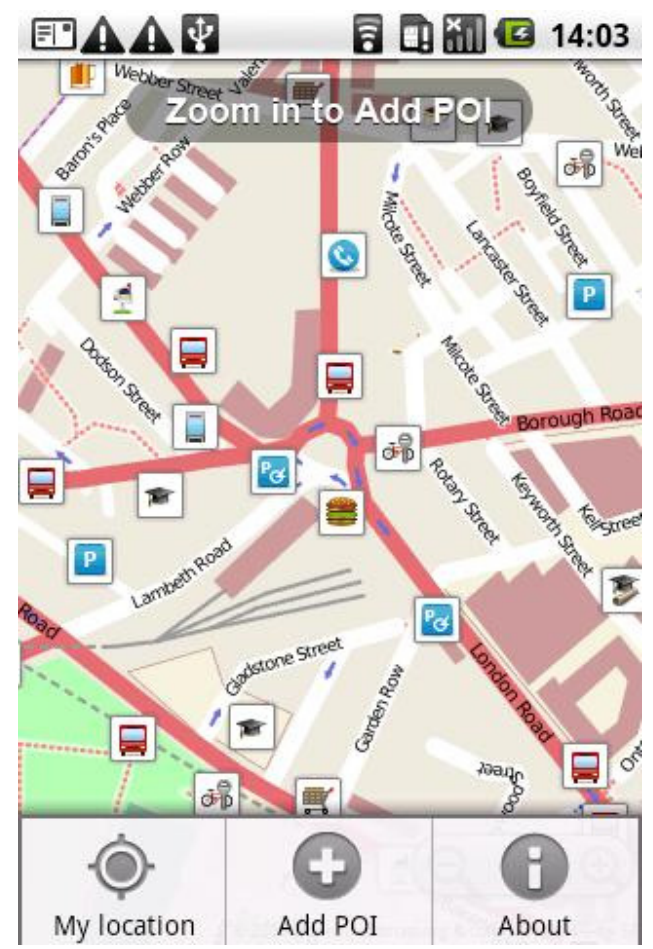

Fig. 5. The Mapzen POI collector for OpenStreetMap. The screenshot shows the application working on an Android mobile phone

rich information about Foursquare venues. The purpose of Foursquare is for friends to share where they are and tell others about the places they request. The whole thing is known as a 'Check-In'. People 'Check-In' to places and they share where they are in addition to adding comments about the place. Like 'the food in this place is amazing' or 'Next time you're in this (Location) you got to visit (Place)'. This could potentially be extended to healthcare facilities or locations. Foursquare is a mobile location game and users can gain points for 'check-ins' to certain locations, repeated visits, etc. After gaining a given number of points for a particular location the user can redeem these points for "freebies" - maybe a free can of soda, hamburger, etc. The mobile applications supporting Foursquare automatically detect location using the integrated GPS on the mobile device. The fourth user-content target is OpenStreetMap. For the mobile user the Mapzen POI Collector [29] allow users of iPhones or Android phones to contribute Points of Interest (POI) to the OpenStreetMap database. A screenshot of Mapzen on an Android phone is shown in Figure 5. The application is free to download and use. The user-interface allow users to choose spatial tagging information from the accepted list of tags for OpenStreetMap or the user can add their own tags. The other options: to upload GPX traces and trails and to edit existing data in the OpenStreetMap database is described above. The common characteristic amongst the four targets for the user content is that the user is not required to possess advanced GIS or spatial/cartographical skills to use these services.

\section{B. Proposed Healthcare Extension to OpenStreetMap}

Currently the amenity key is used for a multitude of unrelated subjects. Some of these subjects relate to healthcare, as outlined in Table I. There are currently two proposals being dicussed within the OpenStreetMap community regarding the addition of keys specifically related to objects describing health care/public health/services. These can be viewed on the OpenStreetMap Wiki pages [30]. The information on these proposals are subject to change. We shall update this paper accordingly at the camera-ready stage if significant changes have taken place between now and then. In one sense the current overloading of keys such as amenity to describe healthcare and other diverse functions works well in OpenStreetMap. There are no requirements that the contributors to OpenStreetMap have very detailed knowledge of the building, facility, or service they are mapping. The expectation is that these contributors will use the accepted tagging ontology provided (as outlined in [31]). As Stvilia and Jorgensen [32] conclude, tagging must always remain an activity the contributors to social media want to carry out. In OpenStreetMap, provided contributors choose tagging from the accepted ontology of tags, all OpenStreetMap map rendering software can interpret the key values and label the output map accordingly. Overloading of the certain keys for health could lead to a situation where the area of health related services are simplified, for tagging purposes, too severely. This new healthcare extension proposal in OpenStreetMap provides support for: tagging objects in a detailed way for support groups, counselling centres, health related services; the real relationships of a hospital and its functions; prevention facilities such as counselling centres (and their area of practice such as drugs, crisis, sexual abuse, or rehabilitation) or community driven services such as support groups. While on first glance this may appear as if greater tagging burden is being placed on contributors to OpenStreetMap the advantages of such a detailed and specific approach are numerous. More advanced types of healthcare applications could be developed based upon the free and open availability of such detailed information

\section{Summary of INTEGRATION OF VGI INTO PERVASIVE HEALTH APPLICATIONS}

In this section we provide a summary of the advantages and disadvantages of integration of VGI into pervasive health applications. This is necessary to allow experts and policy makers from outside the GIS/VGI domain to understand the strengths and weakness of VGI.

\section{A. Advantages of this approach}

- Low Cost: The cost of smartphones, GPS-enabled cameras and phones, Internet access, etc is becoming more competively priced every day.

- Mass participation: The communities which have built around Wikipedia, OpenStreetMap, Flickr, etc are truly massive and are on a global scale. It is impossible to imagine how OSS or VGI would have succeeded without 
the long-distance large-scale collaboration enabled by the Internet and the formation of online communities [33]. At the time of writing (February 2011) statistics on the OpenStreetMap website report 300,000 mappers, up from 200,000 in January 2010.

- Common Interest: Health is a common interest for most people - everyone strives for better health

- Extensive Existing Infrastructure: The VGI projects/options mentioned in this paper: OSM, Flickr, Twitter etc exist and have global hardware infrastructure in place.

- Government Buy-in: In OSM, for example, there are signs of government buy-in throught the donation of government collected or generated spatial data [34]. This includes: CORINE Land Cover data in Europe, the GeoBase project from Canada, LINZ (Land Information New Zealand) in New Zealand and TIGER from the United States census bureau

- Active GIS research area: VGI is a "hot topic" in the GIS community. This field of research is maturing very quickly. There will be opportunities for collaboration with health informatics and take advantage of multidisciplinary approaches.

- Multilingual Options: Multilingual tagging is available in OSM and Flickr, for example. Support is strongest for the naming of geographic objects: streets, buildings, roads, land areas, etc.

- Spatial Coordinates: The geographic datum of OpenStreetMap worldwide is WGS-84. In OSM all spatial data is stored in WGS 84 (Longitude, Latitude) - this is compatible with almost all GPS devices and ensures consistency across national boundaries.

- Open Data License: OpenStreetMap can be used freely under the terms of the Creative Commons AttributionShareAlike 2.0 license. This type of license has "helped promote free culture" [33]. In a nutshell this means that one can basically can do what you like with the data provided the original creator and the license is mentioned. Subsequently anyone else can do the same with anything you produce based on it.

\section{B. Disadvantages of this approach}

In this section we outline a number of disadvantages that must be considered before integrating VGI into pervasive health applications.

- Crowdsourced Community Coordination: There could potentially be issues around coordination of crowdsourced communities. Will members of these communities remain involved and interested in the goals/mission of the project? If there are coordination or management problems of these "virtual communities" data quality or data reliability issues could emerge.

- Urban Bias: In 2008, for the first time in history, more than half of the world's population was living in towns and cities. By 2030 this number will swell to almost 5 billion, with urban growth concentrated in Africa and Asia.

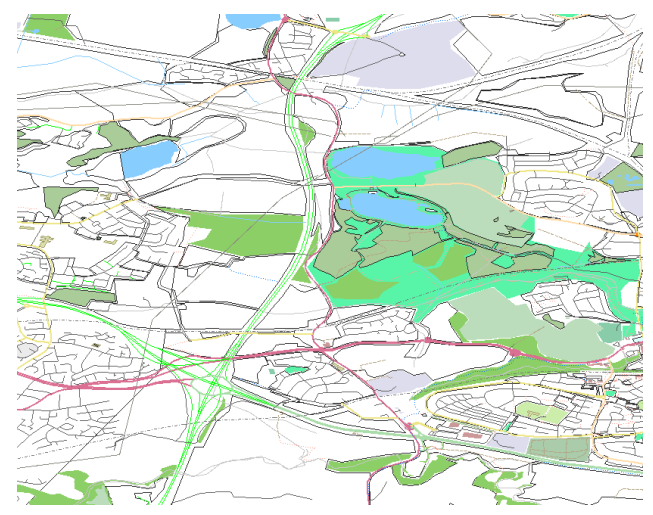

Fig. 6. OpenStreetMap data for Glasgow, Scotland. The spatial data is downloaded directly into the Open Source GIS package QuantumGIS.

While mega-cities have captured much public attention, most of the new growth will occur in smaller towns and cities [35]. Several authors, including Over et al. [36], have reported urban biases in the collection of VGI. This presents a problem for rural areas and communities where such geographic regions may not even be represented in VGI databases. Even within cities this can be an issue as shown in Figure 6 for Glasgow, Scotland. There are many "white spaces" on this rendered map of OpenStreetMap for Glasgow (Feb. 2011).

- No business models: While VGI projects have been successful in generating venture capital and investments (such as Mapquest [4] and Microsoft [5]) there are few, if any, successful business models of companies using an exclusive VGI approach. Part of future related work would include investigating the opportunities in this domain and how the healthcare informatics industry would view the VGI communities.

- Internet access and GPS devices: The "cost" to VGI is the assumption that Internet access is readily available and that contributors own or can access GPS-enabled devices. In Africa in 2010 only $10 \%$ of the population have access to the Internet compared to almost $60 \%$ in Europe [35]. This could create a "digital divide" issue where large geographical areas will remain unmapped in OpenStreetMap, un-photographed in Flickr, etc. This issue could be linked with the coordination of efforts in VGI projects to ensure a more balanced geographical coverage.

In the next section we discussion the important conclusions from this paper and provide some outlook for future work on this topic.

\section{Conclusions And OUtLooK}

To the best of our knowledge linkages between Volunteered Geographic Information (VGI) and pervasive health computing have not been actively explored yet. In the example of Lampos and Cristianini [9] rates of flu occurrences in the UK were determined by text-mining of georeferenced Twitter messages using the open data interface to Twitter. Whilst this 
approach does not give the true rates of flu occurrences the authors conclude that such an approach "give an early warning in various healthcare situations". The crucial aspect of this is that it "mostly can give timely and free information to health agencies to plan health care". All of the information is volunteered. Kostkova et al [37] argue that "the potential of social networking system for early warning systems and for better understanding public concerns about their health is enormous". Similiar examples exist from analysis of Google search statistics. Beyer [38] correlated information extracted from Google Insights for Google Search data with hospital admissions. Beyer found that "Internet search volume activity for kidney stones correlates strongly with temporal and regional kidney stone insurance claims data". Conrad [39] provides similiar results by analyzing influenza-like illness (ILI) data from the U.S. Centers of Disease Control and Prevention, and anonymized, aggregated Google search query data. Conrad concludes that a rise in the frequency of certain influenzarelated search terms in a place corresponds with a rise in actual flu activity for that area.

Historically, both data creation and mapping in the face of disaster required the skills of trained professionals. After the Haitian earthquake, relatively untrained volunteers, NGOs, and citizens were all able to create data critical to the recovery and maps that contextualized this data [16]. VGI played a critical role in the emergency response in Haiti. OpenStreetMap data was heavily used by multiple agencies and NGOs on the ground. Mobile communication devices provide ubiquitous user interfaces for the users (from healthcare professionals to citizens). The possibilities this technology offers for healthcare delivery are vast and the realization process of the potential has only just begun [2]. Using the VGI paradigm as a means of collecting, managing, and disseminating health-care information is a promising research area and worthy of further investigation. The ability to integrate, fuse, or "mash-up" [40] these spatial data with other free and openly available sources of data (such as population statistics, population density, road networks, social deprivation indices, etc) could yield new understandings of issues in relation to provision of healthcare, dissemination of information etc. Citizens are not the only benefactors. The research community will benefit from these data resources being available freely and openly providing them with a new research tool that could help answer questions not previously addressable because of obstacles preventing access to up-to-date and accurate data. Hospitals, physicians, policy makers, and researchers could use these data for benchmarking and to identify targets for improvement efforts [1]. Interoperability with existing health data and software systems will be crucial if the use of web-GIS in public health is to gain acceptance among public health practitioners and the general public [18]. The issue of data quality must also be addressed by the VGI community. The percieved lack of cartographical, surveying, and GIS skills of contributors has seen spatial quality in OSM become a major issue. Mooney et al [41] remark that there are no accepted metrics for measuring the quality of OSM or to a wider extent the quality of VGI. Given the dynamic and organic nature of the spatial data contained in the OSM databases the quality of the spatial data can change considerably quickly [42]. The recent study by Zielstra and Zipf [43] of OSM and TeleAtlas for Germany shows that "while professional data is not without it's faults the coverage of OSM in rural areas is too small to be seriously considered a sophisticated alternative for any applications". We feel that it will be necessary for some forms of data quality control be introduced if VGI or OSM is to be seriously considered for health computing applications.

Clarke et al. [44] see advantages in using VGI/spatial data tools. They comment that using spatial data tools such as Google StreetView available on the Internet can provides health researchers with a "virtual audit instrument for geographical areas which can provide reliable indicators of recreational facilities, the local food environment, and general land use". The use of OpenStreetMap as a "virtual audit instrument" is an exciting application. The dynamic nature of the OpenStreetMap data would provide researchers with a constantly evolving picture of a given local environment. Crucially, Clarke et al. [44] stress that these potential applications can "significantly reduce the costs of collecting data objectively and do so in an unobtrusive manner". Eysenbach [45] stresses that the "open" philosophy of Web 2.0 tools will raise expectations of the "Facebook generation" in terms of dealing with health related data. He comments that "Web 2.0 savvy consumers will push the envelope and demand more than just a health information portal which allows them to view or access their data but not to do anything else with it". He claims that citizens will demand full control over their data with potentially, as a minimum, requirement for an XML export to their mobile or computing device. There is an immediate requirement that the research communities in GIS/VGI and health informatics provide examples of successful integration of VGI and health data/information to assist in validating the approach outlined in this paper.

\section{ACKNOWLEDGMENT}

Dr. Peter Mooney is a research fellow at the Dept of Comp. Sci NUIM and he is funded by the Irish Environmental Protection Agency STRIVE programme (grant 2008-FS-DM14-S4). Dr. Padraig Corcoran is a lecturer and post-doctoral researcher also at the Dept of Comp. Sci NUIM. Dr. Corcoran is part of STRAT-AG which is a Strategic Research Cluster grant (07/SRC/I1168) funded by Science Foundation Ireland under the National Development Plan. The authors gratefully acknowledge this support.

\section{REFERENCES}

[1] P. H. Conway and J. M. VanLare, "Improving access to health care data: The open government strategy," Journal of the American Medical Association (JAMA), vol. 304, no. 9, September 2010.

[2] I. Korhonen and J. Bardram, "Guest editorial introduction to the special section on pervasive healthcare," Information Technology in Biomedicine, IEEE Transactions on, vol. 8, no. 3, pp. 229 -234, 2004.

[3] OpenStreetMap, "The OpenStreetMap project," Online: http://www. openstreetmap.org, February 2011. 
[4] MapQuest, "Aol corporate communications - mapquest 'opens up' in europe with open-source mapping with uk launch," AOL Online Blog report http://corp.aol.com/2010/07/09/ mapquest-opens-up-in-europe-with-open-source-mapping-with-uk-1/, July 2010

[5] Bing Maps, "Bing engages open maps community," Microsoft Bing Maps Online Blog report http://www.bing.com/community/site_blogs/ b/maps/archive/2010/11/23/bing-engages-open-maps-community.aspx, November 2010.

[6] Twitter, "The official Twitter application programming interface (api)," Online: http://dev.twitter.com/doc, February 2011.

[7] Flickr, "The app garden - the official Flickr application programming interface (api)," Online: http://www.flickr.com/services/api/, February 2011.

[8] GeoNames, "TheGeoNames webservices and data export listing," Online: http://www.geonames.org/export/, February 2011.

[9] V. Lampos and N. Cristianini, "Tracking the flu pandemic by monitoring the social web," in Cognitive Information Processing (CIP), 2010 2nd International Workshop on, 2010, pp. $411-416$.

[10] L. Hollenstein and R. Purves, "Exploring place through user-generated content: Using flickr to describe city cores," Journal of Spatial Information Science, vol. 1, no. 1, pp. 21-48.

[11] K. Oyri and P. J. Murray, "osni.info-using free/libre/open source software to build a virtual international community for open source nursing informatics," International Journal of Medical Informatics, vol. 74, no. 11-12, pp. 937 - 945, 2005.

[12] K. Vance, W. Howe, and R. P. Dellavalle, "Social internet sites as a source of public health information," Dermatologic Clinics, vol. 27, no. 2, pp. $133-136,2009$.

[13] A. Georgiou, "Data, information and knowledge: the health informatics model and its role in evidence-based medicine," Journal of Evaluation in Clinical Practice, vol. 8, no. 2, pp. 127-130, 2002.

[14] F. Ormeling, "From ortelius to openstreetmap transformation of the map into a multifunctional signpost," in Cartography in Central and Eastern Europe, ser. Lecture Notes in Geoinformation and Cartography, G. Gartner and F. Ortag, Eds. Springer Berlin Heidelberg, 2010, pp. $1-16$.

[15] M. M. Haklay and P. Weber, "Openstreetmap: User-generated street maps," IEEE Pervasive Computing, vol. 7, no. 4, pp. 12-18, 2008.

[16] M. Zook, M. Graham, T. Shelton, and S. Gorman, "Volunteered geographic information and crowdsourcing disaster relief: A case study of the haitian earthquake," World Medical \& Health Policy, vol. 2, no. 2, p. Article 2, 2010.

[17] C. lvingson, J. Hallberg, T. Timpka, and K. Lindqvist, "Ethical issues in public health informatics: implications for system design when sharing geographic information," Journal of Biomedical Informatics, vol. 35, no. 3, pp. $178-185,2002$.

[18] Q. Yi, R. Hoskins, E. Hillringhouse, S. Sorensen, M. Oberle, S. Fuller, and J. Wallace, "Integrating open-source technologies to build lowcost information systems for improved access to public health data," International Journal of Health Geographics, vol. 7, no. 29, 2008.

[19] B. Janamanchi, E. Katsamakas, W. Raghupathi, and W. Gao, "The state and profile of open source software projects in health and medical informatics," International Journal of Medical Informatics, vol. 78, no. 7, pp. 457-472, 2009.

[20] C. J. McDonald, G. Schadow, M. Barnes, P. Dexter, J. M. Overhage, B. Mamlin, and J. M. McCoy, "Open source software in medical informatics-why, how and what," International Journal of Medical Informatics, vol. 69, no. 2-3, pp. 175-184, 2003, working Conference on Health Information Systems.

[21] D. Greiling, G. Jacquez, A. Kaufmann, and R. Rommel, "Space-time visualization and analysis in the Cancer Atlas Viewer," Journal of Geographical Systems, vol. 7, no. 1, pp. 67-84, 2005.

[22] S. Gao, D. Mioc, F. Anton, X. Yi, and D. Coleman, "Online GIS services for mapping and sharing disease information," International Journal of Health Geographics, vol. 7, no. 8, 2008.

[23] S. Gao, D. Mioc, X. Yi, F. Antonm, E. Oldfield, and D. Coleman, "Towards Web-based representation and processing of health information," International Journal of Health Geographics, vol. 8, no. 3, 2009.

[24] A. MacEachren, S. Crawford, M. Akella, and G. Lengerich, "Design and Implementation of a Model, Web-based, GIS Enabled Cancer Atlas," The Cartographic journal, vol. 45, no. 4, pp. 246-260, 2008.

[25] OpenStreetMap, "Beginners' guide to openstreetmap," OpenStreet-
Map Wiki: http://wiki.openstreetmap.org/wiki/Beginners_Guide, February 2011.

[26] JOSM, "The Java editor for OpenStreetMap," Available for Download from http://josm.openstreetmap.de/, February 2011.

[27] TagInfo, "A webservice query engine for the tags in the openstreetmap database," Online: http://taginfo.openstreetmap.de/, February 2011.

[28] FourSquare, "The FourSquare developer api," Developer Documentation available at http://developer.foursquare.com/, February 2011.

[29] Cloudmade, "The mapzen poi collector for openstreetmap," Information available at http://mapzen.cloudmade.com/mapzen-poi-collector and free-to-download from the Android Marketplace and the iPhone App Store, February 2011.

[30] Fabi, "Proposed features/healthcare 2.0," OpenStreetMap Wiki pages: http://wiki.openstreetmap.org/wiki/Proposed_features/Healthcare_2.0, February 2011.

[31] OSM, "The map features page on openstreetmap.org - the community agreed ontology of tags," Online Wiki Page: http://wiki.openstreetmap. org/wiki/Map_Features (checked February 2011), February 2011.

[32] B. Stvilia and C. Jrgensen, "User-generated collection-level metadata in an online photo-sharing system," Library \& Information Science Research, vol. 31, no. 1, pp. $54-65,2009$.

[33] G. Cheliotis, "From open source to open content: Organization, licensing and decision processes in open cultural production," Decision Support Systems, vol. 47, no. 3, pp. 229-244, 2009, online Communities and Social Network.

[34] OpenStreetMap, "Catalogue of bulk import of datasets to openstreetmap," OpenStreetMap Wiki: http://wiki.openstreetmap.org/wiki/Import/ Catalogue, February 2011.

[35] UNFPA, "State of world population 2009: Facing a changing world: Women, population and climate," Online from United Nations Population Fund http://www.unfpa.org/swp/2009/en/: ISBN 978-0-89714-9587, December 2009.

[36] M. Over, A. Schilling, S. Neubauer, and A. Zipf, "Generating webbased 3d city models from openstreetmap: The current situation in germany," Computers, Environment and Urban Systems, vol. 34, no. 6, pp. 496 - 507, 2010, geoVisualization and the Digital City - Special issue of the International Cartographic Association Commission on GeoVisualization.

[37] P. Kostkova, E. de Quincey, and G. Jawaheer, "The potential of social networks for early warning nad outbreak detection systems: the swine flu twitter study," International Journal of Infectious Diseases, vol. 14, no. Supplement 1, pp. e384-e385, 2010, 14th International Congress on Infectious Diseases (ICID) Abstracts.

[38] B. N. Breyer, S. Sen, D. S. Aaronson, M. L. Stoller, B. A. Erickson, and M. L. Eisenberg, "Use of google insights for search to track seasonal and geographic kidney stone incidence in the united states," Urology, vol. In Press, Corrected Proof, pp. -, 2011.

[39] C. Conrad, "Google flu trends: Mapping influenza in near real time," International Journal of Infectious Diseases, vol. 14, no. Supplement 1 , pp. e185-e185, 2010, 14th International Congress on Infectious Diseases (ICID) Abstracts.

[40] S. Anand, M. Batty, A. Crooks, A. Hudson-Smith, M. Jackson, R. Milton, and J. Morley, "Data mash-ups and the future of mapping," Tech. Rep. Horizon Scanning Report 10-01, November 2010.

[41] P. Mooney, P. Corcoran, and A. C. Winstanley, "Towards quality metrics for openstreetmap," in Proceedings of the 18th SIGSPATIAL International Conference on Advances in Geographic Information Systems, ser. GIS '10. New York, NY, USA: ACM, 2010, pp. 514-517.

[42] _ "A study of data representation of natural features in openstreetmap," in Proceedings of the 6th GIScience International Conference on Geographic Information Science, ser. GIScience 2010. University of Zurich, 2010, p. p150.

[43] D. Zielstra and A. Zipf, "A comparative study of proprietary geodata and volunteered geographic information for germany," in Proceedings AGILE 2010: The 13th AGILE International Conference on Geographic Information Science, M. Painho, M. Y. Santos, and H. Pundt, Eds. Guimarães, Portugal: Springer Verlag, 2010.

[44] P. Clarke, J. Ailshire, R. Melendez, M. Bader, and J. Morenoff, "Using google earth to conduct a neighborhood audit: Reliability of a virtual audit instrument," Health \& Place, vol. 16, no. 6, p. 1224.

[45] G. Eysenbach, "Medicine 2.0: Social networking, collaboration, participation, apomediation, and openness," Journal of Medical Internet Research, vol. 10, no. 3, p. e22, August 2008. 